CONTRIBUCIÓN DEL $3^{\text {ER }}$ CONGRESO LATINOAMERICANO DE MACROINVERTEBRADOS DE AGUA DULCE: BIODIVERSIDAD Y ECOLOGÍA FUNCIONAL EN EL NEOTRÓPICO

\title{
RELACIONES TRÓFICAS DE DOS LAGUNAS EN ÁREAS DE REHABILITACIÓN DENTRO DEL COMPLEJO CARBONÍFERO DEL CERREJON, LA GUAJIRA - COLOMBIA
}

\author{
TROPHIC RELATIONS OF TWO LAGOONS IN AREAS OF REHABILITATION WITHIN OF \\ COAL COMPLEX OF THE CERREJON, LA GUAJIRA - COLOMBIA
}

\author{
Ana Milena Barros-Maestre y Cristian Granados-Martínez
}

\begin{abstract}
RESUMEN
Se determinaron las relaciones tróficas de dos ecosistemas acuáticos (Laguna el Cisne y El muerto) en áreas de rehabilitación dentro del complejo carbonífero el Cerrejón. Para la construcción de las redes tróficas se aplicaron diferentes metodologías para cada componente biológico (fitoplancton, zooplancton, macrófitas, macroinvertebrados, peces, reptiles y aves). Los muestreos se realizaron cada quince días durante dos épocas climáticas (lluvias y sequía). Para determinar los hábitos alimenticios y los gremios tróficos, se utilizó información secundaria en la mayoría de los grupos biológicos, exceptuado a los macroinvertebrados, a los cuales se les analizó el tracto digestivo. Basado en matrices binarias (presencia / ausencia de conexiones), se construyeron las redes tróficas utilizando un modelo estático. Como resultados se establecieron cuatro categorías tróficas en las dos lagunas, las cuales incluyen: omnívoros, depredadores, herbívoros y detritívoros. Entre los principales atributos se resaltan, el número de eslabones que fue de 134 y 226, para las lagunas El Cisne y El Muerto respectivamente, mientras que los valores de conectancia fueron muy cercanos, con 0,23 para El Cisne y 0,22 para El Muerto. El valor de la conectancia en este trabajo fue alto en contraste con otros trabajos realizados en lagunas naturales. Este trabajo es el primero que describe las relaciones tróficas en lagunas artificiales en el Caribe colombiano y servirá como ayuda al conocimiento de las relaciones tróficas de sistemas influenciados por minería.
\end{abstract}

PALABRAS CLAVE: Lagunas Artificiales, Red Trófica, Trofoespecies, Hábitos Alimenticios

\begin{abstract}
We are determine the trophic relationships of two artificial aquatic ecosystems. We studied Cisne and Muerto lagoon’s belonging to areas of rehabilitation land within the Cerrejón coal complex were. We used different methodologies were applied for each biological component (phytoplankton, zooplankton, macrophytes, macroinvertebrates, fish, reptiles and birds). Sampling was done every two weeks during two climatic seasons (rain and drought). To determine dietary habits and trophic guilds, secondary information was used in most biological groups, except for the macroinvertebrates to which the gut contents was analyzed. Based on binary matrices (presence / absence of connections) the trophic networks were constructed using a static model. Four trophic categories were established in the two lagoons, which include: omnivores, predators, herbivores and detritivores. Among the main attributes, the number of links was 134 and 226 for the Cisne and Muerto lagoon respectively. The conectance values were very close, with 0.23 for El Cisne and 0.22 for El Muerto. The value of the connectivity recorded in this work was high in contrast to other works carried out in natural lagoons. This work is the first that describes the trophic relationships in artificial lagoons in the Colombian Caribbean and will serve as an aid to the knowledge of the trophic relationships of systems influenced by mining.
\end{abstract}

KEY WORDS: Artificial lagoons, Food web, Trophospecies, feeding habits 


\section{INTRODUCCIÓN}

Las funciones ecológicas de un ecosistema pueden ser descritas a través de atributos asociados a las especies, que son capaces de reflejar su adaptación a las condiciones ambientales (Townsend y Hildrew, 1994). Las redes tróficas reflejan los hábitos alimenticios y algunas interacciones bióticas que se dan entre los organismos que constituyen una comunidad (Molina y Abarca, 2011), además muestran los patrones de flujos de energía y materia entre los posibles compartimentos dentro de un ecosistema. Frecuentemente estos compartimentos representan "especies tróficas ó trofoespecies" o todas aquellas especies que forman parte de un mismo grupo de presas o de depredadores (Link, 2002).

La estructura trófica no es más que la organización de las especies con base en el alimento que consumen (Valverde et al., 2005). Entre los parámetros más usados para describir dicha estructura, se encuentran: la red trófica, la longitud de la cadena trófica, los niveles tróficos y la conectancia (Warren, 1994; Post, 2002; Valverde et al., 2005). La mayoría de estudios en los ecosistemas acuáticos del Neotrópico, se han enfocado en buscar herramientas biológicas para el diagnóstico de la calidad ambiental del agua, sin tener en cuenta el papel trófico y otros aspectos funcionales y ecológicos (Greathouse y Pringle, 2006). Por ende es necesario que se genere, no solamente información de la estructura, sino también de la función, tanto de los organismos así como de las comunidades que habitan los ecosistemas acuáticos del neotrópico, de esta manera se podrán entender los patrones funcionales a partir de modelos ecológicos (Tomanova et al., 2006; Thompson et al., 2012).

El complejo carbonífero de El Cerrejón tiene como actividad principal la explotación minera desarrollada en superficie a cielo abierto, lo cual afecta drásticamente el paisaje tanto en el sitio de extracción del mineral como en su entorno, causando la pérdida de hábitats, contaminación de suelos, del aire y del agua a causa de las explosiones utilizadas para la remoción del manto terrestre y del mineral (Gualdrón, 2011). El Cerrejón cuenta con diferentes lagunas artificiales distribuidas en todo el complejo carbonífero, entre estas algunas lagunas suplen necesidades de riego en el complejo y otras sirven de áreas de rehabilitación para albergar a fauna reubicada (Gualdrón, 2011).
El análisis de las redes tróficas brinda información de la estructura de los ecosistemas sobre diferentes niveles, a nivel individual, intermedio y grupal. El nivel individual puede describir la posición de una especie dentro de la red, así como su posible efecto sobre otras especies (Ramírez et al., 2010). Dichas relaciones no han sido estudiadas en las lagunas del complejo carbonífero de El Cerrejón, por consiguiente, el objetivo de este estudio fue analizar las relaciones tróficas existentes en las lagunas artificiales El Cisne y El Muerto, pertenecientes al Complejo Carbonífero del Cerrejón a través del conocimiento de hábitos alimenticios de los taxones asociados a las lagunas.

\section{MATERIALES Y METODOS}

\section{Área de estudio}

Las lagunas El Cisne y El Muerto son ecosistemas artificiales diseñados con el fin de ser lugares de reubicación de fauna acuática, en zonas a intervenir 0 en zonas de trabajo del complejo minero. Se encuentran localizadas al norte de Colombia en el departamento de La Guajira, dentro del complejo carbonífero de El Cerrejón. Las zonas de rehabilitación se caracterizan por tener suelos que fueron sujetos a un proceso de rehabilitación con siembra de especies nativas de formación xerofítica tales como Prosopis juliflora, Bulnesia arborea, Platymiscium pinnatum, Cereus repandus, Pereskia guamacho, Borreria essouca, Phytecellobium fortex, Vachellia tortuosa, Tabebuia billbergii, Lonchocarpus puctatus, Mimosa arenosa, Haematoxylum brasiletto y Capparis odoratissima. La laguna El Muerto se localiza entre los $11^{\circ} 06^{\prime} 05^{\prime}$ N y $72^{\circ} 41^{\prime} 27^{\prime \prime}$ W (Figura 1). Posee un área aproximada de $35,14 \mathrm{~km}^{2}$. La laguna El Cisne se ubica entre los $11^{\circ} 05^{\prime}$ $53^{\prime \prime} \mathrm{N}$ y $72^{\circ} 40^{\prime} 39^{\prime \prime} \mathrm{W}$ (Figura 1) y presenta un área aproximada de $4,86 \mathrm{~km}^{2}$.

\section{Recolección de muestras}

Los muestreos se realizaron cada quince días, entre los meses de febrero y abril (época seca) y septiembre y noviembre (época de lluvias). Se aplicaron diferentes metodologías para cada grupo biológico siguiendo los procedimientos estándar de Downing y Rigler (1984). El fitoplancton se recolectó con redes de un poro de 20 $\mu \mathrm{m}$, estas muestras se depositaron en frascos plásticos y se fijaron con lugol (Vicente et al., 2005). Para la recolecta del zooplancton se realizaron arrastres con 
redes con poro de $60 \mu \mathrm{m}$ y frasco colector, las muestras se almacenaron en botellas plásticas fijadas con etanol al 70 \% (Samanez et al., 2014). Los macroinvertebrados se recolectaron con una draga Eckman y una red de mano con ojo de malla de $250 \mu \mathrm{m}$, se guardaron en bolsas plásticas de calibre grueso y se fijaron con etanol al $70 \%$, para su traslado al laboratorio. Para el muestreo de macrofitas se utilizó el protocolo propuesto por Cirujano et al. (2005). Para el muestreo de peces se utilizó una atarraya con ojo de malla de $0,5 \mathrm{~cm}$ y se fijaron en formol al $10 \%$. Los avistamientos de aves y otros vertebrados se desarrollaron con el método de muestreo relevamiento por encuentros visuales (Gallina y López-González, 2011). Para el avistamiento de aves se utilizaron binoculares marca Bushnell 17-5010. La identificación de las aves se realizó con ayuda de la guía de Steven (2001) y la de los reptiles con AngaritaSierra et al. (2013).

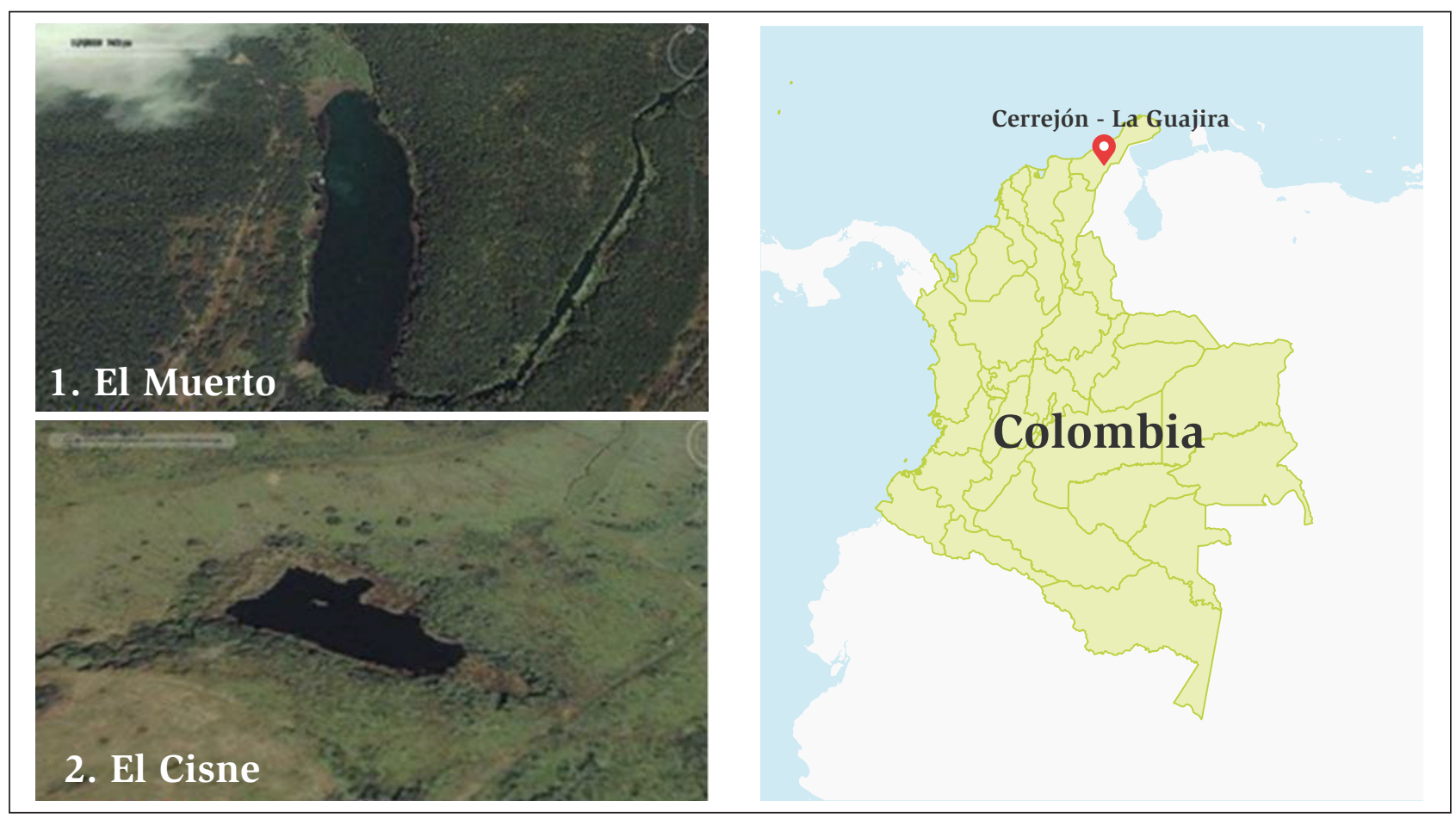

Figura 1. Localización de las lagunas El Cisne y El Muerto. En la parte superior se observa la laguna El Muerto, mientras que en la parte inferior la laguna El Cisne. Tomado y modificado de Google Earth.

\section{Fase de laboratorio}

En el laboratorio, las muestras de fitoplancton y zooplancton fueron observadas con la ayuda de un microscopio de marca Olympus CX21LED e identificadas taxonómicamente al nivel de orden. Las muestras de macroinvertebrados se colocaron en bandejas blancas y con ayuda de pinzas entomológicas se separaron y conservaron en etanol al 70 \%. Para la identificación se utilizó un estereoscopio de marca LED Motic SMZ y las claves especializadas de Fernández y Domínguez (2001). Para los peces, cada individuo recolectado fue identificado hasta el máximo nivel taxonómico posible con ayuda de la clave de Jiménez-Segura et al. (2014).
Para determinar los hábitos alimenticios y gremios tróficos a los que pertenecen los macroinvertebrados, se analizó el tracto digestivo de los macroinvertebrados siguiendo la metodología utilizada por Tomanova et al. (2006), donde los tractos de los macroinvertebrados fueron removidos y dispuestos en placas para su observación y determinación de los hábitos utilizando un microscopio.

Para determinar los gremios tróficos (Tabla 1 y 2 ) de todos los organismos se utilizó información secundaria basada en los siguientes autores Oliveros y Rossi (1991); De la Ossa y Riaño (1999); Martínez (1999); Armúa de Reyes (2002); Ordóñez (2002); Alessio et 
al. (2005); Cala (2005); Forero et al. (2006); Tomanova et al. (2006); Andramunio-Acero et al. (2008); Dario (2008); Gómez (2008); Alba (2010); Chará-Serna et al.
(2010); Cumana-Campos (2010); Giraldo-Cañas (2013); Granados-Martínez (2013); Torres-Bejarano (2013); Workiyie y Abebe (2015).

Tabla 1. Listado de los grupos, gremios y taxones encontrados en la laguna El Cisne.

\begin{tabular}{|c|c|c|}
\hline Grupo & Gremio & Taxón \\
\hline \multirow{3}{*}{ Fitoplancton } & \multirow{3}{*}{ Organismos Productores } & Chlorophyceae \\
\hline & & Bacillariophyceae \\
\hline & & Zygnematophyceae \\
\hline \multirow{2}{*}{ Perifiton } & \multirow{2}{*}{ Organismos productores } & Cyanophyceae \\
\hline & & Chrysophyceae \\
\hline Macrofitas & Productores & Paspalum repens, Ludwigia. Helminthorrhiza. \\
\hline \multirow{6}{*}{ Zooplancton } & \multirow{4}{*}{ Z1(Herbívoro) } & Daphnia \\
\hline & & Moina \\
\hline & & Diaphanosoma \\
\hline & & Brachionus \\
\hline & \multirow{2}{*}{ Z2(Herbívoro) } & Diaptomus \\
\hline & & Cyclops \\
\hline \multirow{5}{*}{ Macroinvertel } & \multirow{2}{*}{ M1(Depredador de insectos ) } & Belostomatidae \\
\hline & & Calopterygidae \\
\hline & M2(Depredador de Chironomidaes) & Tanypodinae \\
\hline & M3(Detritívoro) & $\begin{array}{l}\text { Planorbidae } \\
\text { Chironominae } \\
\text { Orthocladiinae }\end{array}$ \\
\hline & & Thiariidae \\
\hline \multirow{2}{*}{ Aves } & A1(Depredador Insectívoro) & Certhiaxis cinnamomeus \\
\hline & A2(Depredador Piscívoro) & Megaceryle torquata \\
\hline \multirow{2}{*}{ Peces } & P1(Hervivoro ) & $\begin{array}{l}\text { Oreochromis niloticus } \\
\text { Hypostomus hondae }\end{array}$ \\
\hline & P2( Depredador Carnívoro Insectivoro) & Aequidens pulcher \\
\hline \multirow{2}{*}{ Reptil } & B(Depredador carnivoro) & Caiman crocodilus fuscus \\
\hline & I(Omnívoro) & Trachemys callirostris \\
\hline
\end{tabular}


Tabla 2. Listado de los grupos, gremios y taxones encontrados en la laguna El Muerto.

\begin{tabular}{|c|c|c|}
\hline Grupo & Gremio & Taxón \\
\hline \multirow{3}{*}{ Fitoplancton } & \multirow{3}{*}{ Organismos productores } & Ceratium \\
\hline & & Chlorophyceae \\
\hline & & Zygnematophyceae \\
\hline \multirow{3}{*}{ Perifiton } & \multirow{3}{*}{ Organismos Productores } & Cyanophyceae \\
\hline & & Chrysophyceae \\
\hline & & Euglenophyceae \\
\hline Macrofitas & Productores & Paspalum repens, Ludwigia helmintorrhiza \\
\hline \multirow{5}{*}{ Zooplancton } & \multirow{4}{*}{ Z1(Herbívoro) } & Daphnia \\
\hline & & Diaphanosoma \\
\hline & & Moina \\
\hline & & Brachionus \\
\hline & Z2(Herbívoro) & Cyclops \\
\hline \multirow{5}{*}{ Macroinvertebrados } & & Thiariidae \\
\hline & M1(Detritívoro) & Planorbidae \\
\hline & & Chironominae \\
\hline & M2(Depredador de Chironomidae) & Tanypodinae \\
\hline & M2(Depredador) & \\
\hline \multirow{8}{*}{ Aves } & & Phalacrocorax brasilianus \\
\hline & A1(Depredador Piscívoro) & Ardea alba \\
\hline & & Anhinga anhinga \\
\hline & A2(DepredadorPicivoro e Insectivoro) & Busarellus nigricollis \\
\hline & & Pilherodius pileatus \\
\hline & A3(Depredador Piscívoro) & Megaceryle torquata \\
\hline & & Ardea herodias \\
\hline & A4(Depredador Insectívoro) & Jacana jacana \\
\hline \multirow{7}{*}{ Peces } & & Cyphocharax magdalenae \\
\hline & P1(Detritívoro Picivoro) & Curimata mivartii \\
\hline & & Hypostomus hondae \\
\hline & & Ctenolucius hujeta \\
\hline & P2(Depredador Picivoro e insectivoro) & Hoplias malabaricus \\
\hline & & Caquetaia kraussii \\
\hline & P3(Omnívoro) & Astyanax fasciatus \\
\hline \multirow{3}{*}{ Reptiles } & B(Depredador) & Caiman crocodilus fuscus \\
\hline & & \\
\hline & I(Omnívoro) & Trachemys callirostris \\
\hline
\end{tabular}




\section{Análisis de los datos}

Basado en matrices binarias (presencia / ausencia de conexiones) se construyeron las redes tróficas utilizando un modelo estático, donde se registraron cuales taxones dentro del sistema se alimentan de otros y las interacciones tróficas que pueden estar presentes o ausentes entre cada par de taxones (AndradeLópez, 2012). Para la realización de los modelos y sus características se aplicó el paquete Package 'foodweb' versión 1-0 de Agosto 29, 2013 (Perdomo, 2013) con ayuda del programa R. versión libre 2.12.2.

\section{RESULTADOS}

\section{Características de las redes tróficas}

La red trófica construida para la laguna El Cisne, muestra 14 trofoespecies, constituida por una especie superior B (Caiman crocodilus fuscus), 10 trofoespecies intermedias y 3 grupos basales (perifiton, fitoplancton y macrófitas) (Figura 2). Presenta dos casos de canibalismo representados por $Z 2$ (zooplancton) y M2 (macroinvertebrados depredadores) y un caso de omnivoría representado por un reptil I (Trachemys callirostris), en la laguna El Muerto, se encontraron 16 trofoespecies, una especie superior B (Caiman crocodilus fuscus), 3 (tres) grupos basales (perifiton, fitoplancton y macrófitas) y 12 trofoespecies intermedias; de los cuales, se evidenciaron dos especies omnívoras representadas por I (Trachemys callirostris) y P3 (Astyanax fasciatus) y 4 (cuatro) casos de canibalismo representados por Z2 (zooplancton), M2 (macroinvertebrados depredadores), P2 (peces depredadores) y P3 (peces omnívoros) como se evidencia en la figura 3.

En la tabla 3, se observan los atributos de las redes tróficas, entre los que se destacan: el número de eslabones el cual fue de 134 en El Cisne y de 226 en El Muerto (Figuras 2 y 3). Por otro lado, Los valores de conectancia fueron muy similares en los dos sistemas con registros de 0,23 y 0,22 en El Cisne y El Muerto respectivamente (Tabla 3). En El Cisne, el $7 \%$ de sus trofoespecies se categorizaron como tope, el $67,4 \%$ como intermedias, el $20 \%$ como basales y el $7 \%$ omnívoras. En la laguna el Muerto los valores fueron muy similares siendo el $6 \%$ trofoespecies tope, $67 \%$ trofoespecies intermedias, $17 \%$ trofoespecies basales y $11 \%$ omnivoras (Tabla 3).

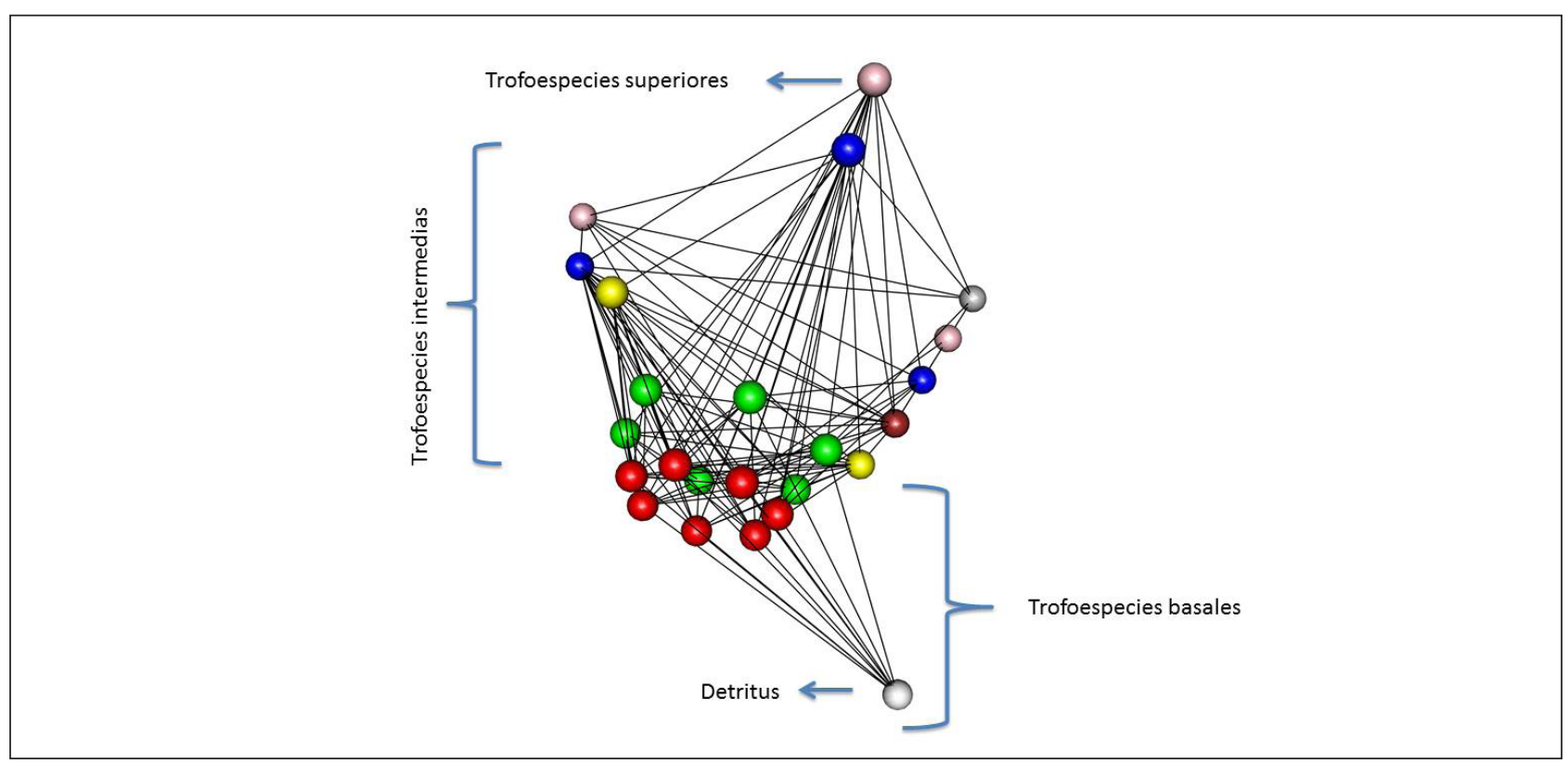

62 Figura 2. Red trófica de la laguna artificial El Cisne. 


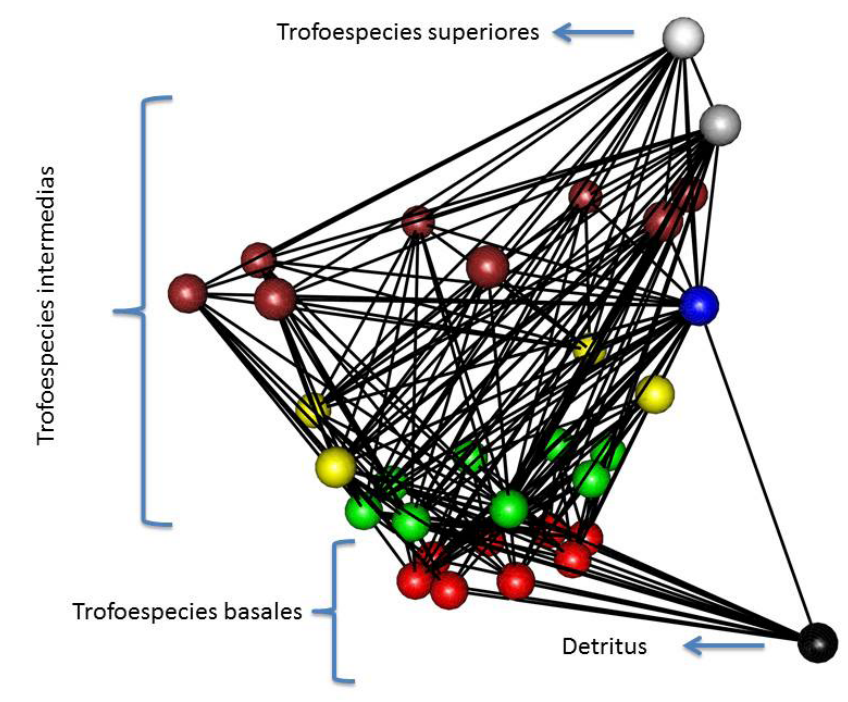

Figura 3. Red trófica de la laguna artificial El Muerto.

Tabla 3. Características de la red trófica de las lagunas El Cisne y El Muerto.

\begin{tabular}{|c|c|c|c|c|c|}
\hline Atributo & El Cisne & El Muerto & Atributo & El Cisne & El Muerto \\
\hline Eslabones & 134 & 226 & \multirow{2}{*}{$\mathrm{N}^{\circ}$. Trofoespecies basales } & \multirow[b]{2}{*}{3} & \\
\hline Conectancia & 0,23 & 0,22 & & & 3 \\
\hline Número de Taxones & 24 & 32 & $\mathrm{~N}^{\circ}$. Omnívoros & 1 & 2 \\
\hline Trofoespecies & 14 & 16 & Frac de T. superiores & 0,04 & 0,03 \\
\hline Densidad de Uniones & 5,58 & 7,05 & Frac de T. intermedias & 0,91 & 0,93 \\
\hline Tamaño Max. De la Red & 4 & 4 & Frac de T. basales & 0,04 & 0,03 \\
\hline \multirow[t]{2}{*}{$\begin{array}{l}\mathrm{N}^{\circ} \text {. Trofoespecies } \\
\text { superiores }\end{array}$} & 1 & 1 & Frac de omnivoría & 0,54 & 0,62 \\
\hline & & & Frac de canibalismo & 0,20 & 0,09 \\
\hline $\begin{array}{l}\mathrm{N}^{\circ} \text {. Trofoespecies } \\
\text { intermedias }\end{array}$ & 10 & 12 & Frac de herbívoros & 0,29 & 0,25 \\
\hline
\end{tabular}




\section{DISCUSIÓN}

El número de taxones encontrados en este estudio difiere con lo reportado por Andramunio-Acero y Caraballo (2012), en un lago de inundación de la Amazonia Colombiana, quienes registraron 75 taxones en contraste con los 40 taxones encontrados en las dos lagunas. Respecto al tamaño de las redes ambas presentaron cuatro niveles tróficos incluyendo productores primarios y consumidores de primer, segundo y tercer orden, lo cual concuerda con lo registrado para las redes tróficas acuáticas, las cuales varían de tres a cuatro (Pimm y Kitching, 1987). El número de conexiones obtenidas en las redes fue de 134 para El Cisne y 226 para El Muerto. Andramunio-Acero y Caraballo (2012) encontraron en su trabajo 40 conexiones teniendo un mayor número de trofoespecies. Lo cual podría indicar que el número de conexiones no depende del número de trofoespecies. El valor de la conectancia registrado en este trabajo fue alto en contraste con el encontrado por Molina y Abarca (2011), en una zona costera y AndramunioAcero y Caraballo (2012) en un lago de la Amazonía Colombiana, pero fue similar al registrado por Cruz et al. (2013) en la Bahía Magdalena de baja California sur, y bajo al obtenido por Alcorlo (2004) respectivamente en los lagos salinos temporales El Piñol y La Muerte de Los Monegros (Zaragoza, España), quienes detectaron valores de 0,2 y 0,50. Lo anterior indica que a pesar de estar enfrentados a ecosistemas de naturaleza artificial, las relaciones tróficas en el sistema para el momento del estudio, fueron muy fuertes, por lo cual se puede considerar que estas lagunas podrían ser más resistentes a la pérdida de taxones o a remoción de individuos comparado con redes con conectancia bajas $(\sim<0,15)$ las cuales son más sensibles a la pérdida de organismos (Estrada, 2007).

Es muy importante destacar finalmente la construcción de las redes tróficas de sistemas de lagunas artificiales como un aporte inicial de este tema en el Caribe colombiano y como ayuda al conocimiento de las relaciones tróficas en ambientes artificiales de agua dulce, influenciados por la minería. Sin embargo, también se muestra la necesidad de realizar estudios en periodos más prolongados de tiempo, con mayor recolecta de información y muestreos que revelen de forma completa la variación de estas relaciones. Es muy importante destacar finalmente la construcción de las redes tróficas de sistemas de lagunas artificiales como un aporte inicial de este tema en el Caribe colombiano y como ayuda al conocimiento de las relaciones tróficas en ambientes artificiales de agua dulce, influenciados por la minería. Sin embargo, también se muestra la necesidad de realizar estudios en periodos más prolongados de tiempo, con mayor recolecta de información y muestreos que revelen de forma completa la variación de estas relaciones.

\section{AGRADECIMIENTOS}

A Pablo Gutiérrez-Fonseca por la construcción de las redes. A Lina Baez por la logística proporcionada para la toma de muestras. También a los integrantes del grupo de investigación EBET de la Universidad de La Guajira quienes ayudaron en las actividades de laboratorio.

\section{BIBLIOGRAFÍA}

Alba, P. 2010. Productividad primaria y estructura de la comunidad fitoplanctónica y su relación con la dinámica hidrológica en el sistema de lagos Yahuarcaca. Tesis de Maestría en Ciencias Ambientales, Universidad Jorge Tadeo Lozano, Bogotá, Colombia.

Alcorlo, P. 2004. Las redes tróficas en las lagunas salinas temporales de Los Monegros (Zaragoza, España). Ecosistemas 13(2): 37-51.

Alessio, V., Beltzer, A., Lajmanovich, R. y Quiroga, M. 2005. Ecología alimentaría de algunas especies de Passeriformes (Furnariidae, Tyrannidae, Icteridae y Emberizidae): consideraciones sobre algunos aspectos de nicho ecológico. Miscelánea 14: 441-482.

Andrade-López, J. 2012. Estructura de las tramas tróficas en ríos neotropicales: Consideraciones metodológicas en el análisis de atributos y procesos. Académica Española, España.

Andramunio-Acero, C. y Caraballo, P. 2012. Análisis de las relaciones tróficas en un lago de inundación de La Amazonia Colombiana. Revista Colombiana de Ciencia Animal 4(1): 102-120.

Andramunio-Acero, C., Duque, S. y Sierra, C. 2008. Estudio sucesional-espacial de la comunidad fitoperifítica asociada a Paspalum repens en el lago Tarapoto (Amazonía Colombiana). Revista de Ciencias 12: 57-72.

Angarita-Sierra, T., Ospina-Sarria, J., Anganoy-Criollo, M., Pedroza-Banda, R. y Lynch, J.D. 2013. Guía de campo de los Anfibios y Reptiles del departamento de Casanare (Colombia). Serie Biodiversidad para la Sociedad, Bogotá-Arauca. 
Armúa de Reyes, A.C. 2002. Sistemática, Aspectos Poblacionales y Asociaciones Interespecíficas de Belostomatidae (Hemíptera) en ambientes acuáticos de la provincia de Corrientes. Tesis doctoral, Facultad de Ciencias Exactas y Naturales y Agrimensura, Universidad Nacional del Nordeste, Argentina.

Cala, P. 2005. Diet of Astyanax Fasciatus and Cyphocharax Magdalenae (Pisces: Characiformes), In The Betania Reservoir, Upper Part Of The Rio Magdalena System, Colombia. Dahlia-Revista de la Asociación Colombiana de Ictiología 8: 3-7.

Chará-Serna, A., Chará, J., Zúñiga, M., Pedraza, G. y Giraldo, L. 2010. Clasificación trófica de insectos acuáticos en ocho quebradas protegidas de la ecorregión cafetera colombiana. Universitas scientarium 15(1): 27-36.

Cirujano, S., Cambra, S. y Gutierrez, C. 2005. Protocolos de muestreo y análisis para macrófitas, Metodología para el establecimiento del Estado Ecológico según la Directiva Marco del Agua. Confederación Hidrográfica del Ebro con la asistencia técnica de URS, Zaragoza, España.

Cruz, V., Morales, M., Navia, A., Arguez, J. y Monte, P. 2013. Análisis funcional de la red trófica de Bahía Magdalena Baja California Sur, México. Latin American Journal of Aquatic Research 41(3): 519-544.

Cumana-Campos, L.J. 2010. Clave para especies de Ludwigia L. (Onagraceae) de la región nor-oriental e insular de Venezuela depositadas en el herbario IRBR. Acta Botánica Venezuelica 33(2): 299-327.

Dario, F. 2008. Estrutura trófica da avifauna em fragmentos florestais na Amazônia Oriental. Con Scientiae Saúde 7(2): 169-179.

De La Ossa, J. y Riaño, R. 1999. Guía para el manejo y cría de la hicotea o jicotea Trachemys scripta callirostris (Gray). Serie Ciencia y Tecnología, Convenio Andrés Bello, Santa Fe de Bogotá.

Downing, J.A. y Rigler, F.H. 1984. A Manual on Methods for the Assessment of Secondary Productivity in Fresh Waters. Second edition, Blackwell Scientific Publications, Gran Bretaña.

Estrada, E. 2007. Food webs robustness to biodiversity loss: the roles of connectance, expansibility and degree distribution. Journal of theoretical biology 244(2): 296-307.

Fernández, H.R. y Domínguez, E. 2001. Guía para la determinación de los artrópodos bentónicos sudamericanos. Universidad Nacional de Tucumán, Tucumán, Argentina.
Forero, M.G., Castaño, M.O. y Rodríguez, M. 2006. Ecología De Caiman crocodilus fuscus en San Andrés Isla, Colombia: Un estudio preliminar. Caldasia 28(1): 115-124.

Gallina, S. y López-González, C. 2011. Manual de técnicas para el estudio de la fauna. Volúmen I. Universidad Autónoma de Querétaro-Instituto de Ecología, A. C., Querétaro, México.

Giraldo-Cañas, D. 2013. Las gramíneas en Colombia: Riqueza, distribución, endemismo, invasión, migración, usos y taxonomías populares. Biblioteca José Jerónimo Triana. Instituto de Ciencias Naturales, Universidad Nacional de Colombia, Bogotá, D.C.

Gómez, M. 2008. Dinámica espacial y temporal de la comunidad fitoplanctónica en el lago Yahuarcaca, Planicie de inundación del río Amazonas. Tesis de Maestría, Pontificia Universidad Javeriana, Bogotá, Colombia.

Granados-Martínez, C. 2013. Análisis de la dieta de los macroinvertebrados bentónicos en un gradiente altitudinal de la cuenca del Rio Gaira (Sierra - Nevada de Santa MartaColombia). Tesis de maestría, Universidad del Zulia, Maracaibo.

Greathouse, E.A. y Pringle, C.M. 2006. Does the river continuum concept apply on a tropical island? Longitudinal variation in a Puerto Rican stream. Canadian Journal of Fisheries and Aquatic Sciences 63: 134-152.

Gualdrón, R.A. 2011. Cerrejon, hacia la rehabilitación de las tierras intervenidas por la minería a cielo abierto. Bogotá, Colombia.

Jiménez-Segura, L.F., Álvarez, J., Ochoa, L.E., Loaiza, A., Londoño, J.P., Restrepo, D., Aguirre, K., Hernández, A., Correa, J.D. y Jaramillo-Villa, U. 2014. Guía Ilustrada Peces Cañón del río Porce, Antioquia. EPM. Universidad de Antioquia, Herbario Universidad de Antioquia - Medellín, Colombia.

Link, J. 2002. Does food web theory work for marine ecosystems? Marine Ecology Progress Series 230: 1-9.

Martínez, G. 1999. Estrategias de alimentación de tres especies del zooplancton límnico (Cladócera). Revista Chilena de Historia Natural 72: 671-676.

Molina, F. y Abarca, L. 2011. Estructura trófica de la zona costera de Alvarado Veracruz, y su relación con la riqueza de trofoespecies. Tesis de Pregrado, Universidad del Mar Campus Puerto Ángel, Puerto Ángel Oaxaca-Mexíco.

65

Oliveros, O. y Rossi, L. 1991. Ecología trófica de Hoplias malabaricus malabaricus (Pisces - Erythrinidae). Revista de la Asociación de Ciencias Naturales del Litoral 22(2): 55-68. 
Ordóñez, J. 2002. Estructura de la comunidad de algas epifíticas en dos macrófitos (Paspalum repens y Polygonum densiflorum) en los lagos de Yahuarcaca (Amazonas Colombia). Tesis de Maestría, Universidad de los Andes, Bogotá, Colombia.

Perdomo, G. 2013. Package 'foodweb'. Version 1-0. August 29, 2013.

Pimm, S. y Kitching, R. 1987. The determinants of food chain lenghts. Oikos 50(3): 302-307. Doi: 10.2307/3565490.

Post, D. 2002. The long and short of food-chain length. Trends in Ecology and Evolution 17: 269-277.

Ramírez, D., Abarca, L., Valero, E. y Macswiney, C. 2010. Redes tróficas, una herramienta de estudios ecosistémicos. Aleph Zero 57: 32-43.

Samanez, I., Rimarachin, V., Palma, C., Arana, J., Ortega, H. y Correa, V. 2014. Métodos de colecta, identificación y análisis de comunidades biológicas: plancton, perifiton, bentos (macroinvertebrados) y necton (peces) en aguas continentales del Perú.- Lima. Ministerio del Ambiente, Perú.

Steven, H.L. y Brown, W.L. 2001. Guía de las aves de Colombia. Princeton University Press, New Jersey.

Tomanova, S., Goitia, E. y Helešic, J. 2006. Trophic levels and functional feeding groups of macroinvertebrates in Neotropical streams. Hydrobiology 56: 251-264.
Thompson, R.M., Brose, U., Dunne, J.A., Hall, R.O. Jr., Hladyz, S., Kitching, R.L., Martinez, N.D., Rantala, H., Romanuk, T.N., Stouffer, D.B. y Tylianakis, J.M. 2012. Food webs: reconciling the structure and function of biodiversity. Trends in Ecology and Evolution 27: 689-697.

Torres-Bejarano, A. 2013. Ecología trófica y dinámica del zooplancton en dos lagos de inundación de la amazonia colombiana. Tesis de Maestría, Universidad Nacional de Colombia, Sede Amazonia.

Townsend, C. y Hildrew, A.G. 1994. Species traits in relation to a habitat templet for river systems. Freshwater Biology 31: 265-275.

Valverde, T., Cano, J., Meave, A. y Carabias, J. 2005. Ecología y medio ambiente. Pearson Educación, México.

Vicente, E., De Hoyos, C., Sanches, P. y Cambra, J. 2005. Protocolos de muestreo y análisis para fitoplacton, Metodología para el establecimiento del Estado Ecológico según la Directiva Marco del Agua. Confederación Hidrográfica del Ebro con la asistencia técnica de URS, Zaragoza, España.

Warren, P.H. 1994. Making connections in food webs. Trends in Ecology and Evolution 9: 136-141.

Workiyie, A. y Abebe, G. 2015. The food and feeding ecology of Nile tilapia, Oreochromis niloticus, in Lake Hayq, Ethiopia. International Journal of Fisheries and Aquatic Studies 2(3): 176-185.

Para citar este artículo: Barros-Maestre, A.M. y Granados-Martínez, C. 2016. Relaciones tróficas de dos lagunas en áreas de rehabilitación dentro del complejo carbonífero del Cerrejon, La Guajira - Colombia. Revista Intropica Vol. 11: 57 - 66 